\title{
Amphotericin B and invasive aspergillosis - how do the data guide us?
}

The escalating incidence of invasive aspergillosis (IA) in immunocompromised patients coupled to the staggering overall case fatality ratio in excess of $50 \%[1,2]$ reminds us that our therapeutic outcomes are suboptimal. Conventional amphotericin $\mathrm{B}(\mathrm{CAB})$ is toxic, which limits compliance. In an attempt to control severe systemic reactions, patients are exposed to potentially harmful opiates, corticosteroids, dopamine and subtherapeutic antifungal dosing. In a recent study, $30 \%$ of 707 patients treated with $\mathrm{CAB}$ developed acute renal failure, resulted in a three-fold excess mortality, 8 days extra hospitalisation and an increased adjusted health care cost of 30000 US dollars per patient [3]. Despite the repeated documentation of such facts, hospital purchasing departments think purely in terms of acquisition costs when budgeting for antifungal agents. Yet amphotericin $B$ has been dubbed the emperor of antifungal drugs - an allusion largely founded on its excellent in-vitro activity.

Therefore, in keeping with many contemporary royals, amphotericin B is changing its habits. The liposomal preparation ( $\mathrm{LAB}$; trade name AmBisome) has the lowest incidence of infusion-related adverse events. Nephrotoxicity is reduced dramatically from around $80 \%$ to $5 \%$. It makes LAB currently the strongest candidate among the three commercially available lipid formulations of amphotericin B (the other two being ABLC and ABCD). Clinical trials and a Cochrane Review of all the lipid-soluble formulations [4] confirm this opinion.

The contentious issues of initiation and dosing quantitation, efficacy [5] and repeated concerns over economics remain. Debates over the design of clinical trials are well known and rehearsed - heterogeneity of patients, variable case definitions, etc. - and will not be repeated here. A recent systematic review of the literature updating the case fatality rates in IA concluded that the mortality of this condition remained high despite the newer formulations of amphotericin B [2]. That review discarded 172 of 222 papers and even the 50 remaining ones had high heterogeneity. Any benefit of lipid formulations would be lost in the jungle of papers. So we should look more carefully at statements such as 'our data suggest a trend for lower casefatality rates with lipid formulations of amphotericin B' [2].
Controlled animal models provide one perspective for this exercise. There is strong evidence for a substantially superior effect of lipid preparations over CAB; e.g., Allende et al. [6] demonstrated a two-fold increased survival with ABCD $1-5 \mathrm{mg} / \mathrm{kg} /$ day, Leenders et al. [7] showed a 2-4-fold decrease in dissemination of Aspergillus with LAB $1-10 \mathrm{mg} / \mathrm{kg} /$ day. Open-label salvage studies confirm a parallel human phenomenon in that patients failing treatment with $\mathrm{CAB}$ responded to the various lipid preparations [5]. Controlled studies also support the impression of heightened efficacy for $\mathrm{LAB}$ over $\mathrm{CAB}$ : improved resolution of fever in patients with antibiotic unresponsive neutropenic fever [8], reduction in breakthrough fungal infections [9], and a better response in patients with fungal infections [10]. The human clinical literature is biased towards the larger experience with $\mathrm{LAB}$; controlled data supporting superior clinical performance of $\mathrm{ABLC}$ and $\mathrm{ABCD}$ over $\mathrm{CAB}$ has yet to be demonstrated convincingly. Furthermore, no convincing head-to-head clinical efficacy comparison trials of any of the lipid preparations have yet been performed, but one toxicity study showed substantially and significantly increased safety of LAB over ABLC [11].

What dose to use - a maximum tolerated or a minimum effective? Are these the same? Here animal studies reveal a non-parallelism $[6,7,12]$. Although bigger doses (over a range $1-10 \mathrm{mg} / \mathrm{kg}$ /day) of either ABCD or LAB sterilise Aspergillus-infected tissues better, this has not translated into clinically meangingful improved survival figures. For example, in Allende's model survival was $55-64 \%$ for $1 \mathrm{mg}$ and $5 \mathrm{mg}$ of $\mathrm{ABCD}$ [6] (and was actually reduced to $<30 \%$ at $10 \mathrm{mg}$ ). This mycologic-survival dissociation phenomenon suggests that factors other than the dosages used impact on survival. The first published prospective randomised clinical efficacy trial of antifungal agents in IA showed no apparent difference in survival when either $1 \mathrm{mg}$ or $4 \mathrm{mg}$ of LAB was used [13]. Heterogeneity of patient characteristics was a characteristic feature of this study, and although both treatment arms were balanced in that regard, the overall analysis could not even detect a trend in favour of the higher dose. However, more patients in a small subgroup with definite IA responded and survived with the higher dose of LAB, suggesting that a better response with a higher dose could be possible. An 
alternative explanation for the apparent outcome equivalence is that dosages in excess of $4 \mathrm{mg}$ are needed to optimise response. This alternative explanation has some support from studies in neutropenic mice with candidosis, where survival was increased from $40 \%$ with $5 \mathrm{mg}$ to $100 \%$ at the maximum tolerated dose of $29 \mathrm{mg}$ [14].

There are other pertinent observations which support both the use of higher doses of LAB and administering $\mathrm{LAB}$ at the earliest stage in the clinical evolution of IA. It is generally accepted that both $\mathrm{CAB}$ and $\mathrm{LAB}$ have concentration-dependent antifungal activity, and this is linked to clinical efficacy [15]. MICs of LAB for several Aspergillus species range up to $2.5 \mathrm{mg} / \mathrm{L}$ $[16,17]$. Steady-state plasma concentrations indicate that even at a dose of $1 \mathrm{mg}$, the $\mathrm{C}_{\max }$ is $12.2 \mathrm{mg} / \mathrm{L}$ [18]. However, the more relevant pharmacokinetic parameter is likely to be the lung tissue concentration. At $1 \mathrm{mg}$ of $\mathrm{LAB}$ that is only $1.8 \mathrm{mg} / \mathrm{kg}$ and this measurement was made in healthy tissue [17]. The levels will be lower in infected tissue, and below the MICs. However, at $5 \mathrm{mg}$ the level is $10.3 \mathrm{mg} / \mathrm{kg}$ [17]. Superior antimycological activity of LAB at higher doses has already been alluded to earlier in this article. Treatment failure has been linked to the poor tissue penetration of amphotericin $B$ into infected lung tissue. In one study the level of $\mathrm{CAB}$ in infected tissue was three times lower $(0.2 \mathrm{mg} / \mathrm{kg})$ than in healthy lung tissue, in a patient who died from IA [19]. This strongly suggests that poor tissue penetration may result in treatment failure. Histopathological studies reveal that the IA lesions are lung infarcts secondary to the vascular lesions, producing the central necrotic nodules surrounded by a haemorrhagic peripheral ring. Antifungal drug penetration is compromised in such tissue. These early nodular lesions, visible on CT scanning [20], rapidly progress to larger fungal masses such as the wedge-shaped pleural-based lung infarcts, cavitary masses and abscesses, confounding the problem of effective drug delivery.

Early and serial computed tomography (CT) scanning is a highly effective modality for diagnosing invasive pulmonary aspergillosis in neutropenic patients [20]. Galactomannan determination is becoming another important early diagnostic tool. Early diagnosis is linked to better survival because amphotericin B treatment is started earlier [21], at a time when fungal burden is relatively small. At some variance with IDSA guidelines [22], many clinical mycologists would give treatment at the earliest possible stage of IA with a dose which would provide a concentration at an infected site in excess of the MIC. LAB at a dose of $\geqslant 5 \mathrm{mg} / \mathrm{kg}$ should be capable of meeting this expectation because there would be small chance of dose interruption, no need for test dosing or gradual incremental dose increasing, and assurance that the drug physically homes in on the site of fungal disease [23].
Support for such a strategy will not be found in the large meta-analysis type of reviews. It will come from reports from individual institutions. We recently reviewed our experience in Tawam Hospital, the UAE's tertiary referral centre for leukaemia, following adoption of a linked policy of early diagnosis and administering modestly high doses of LAB. There were 21 cases of IPA over the last 2 years in patients with chemotherapy-induced neutropenia; 20 of the 21 patients had halo signs. LAB was given at a mean daily dose of $4.4 \mathrm{SD} 2.2 \mathrm{mg} / \mathrm{kg}$, starting within 1 week of ARNF, and for a mean cumulative dose of $5.7 \mathrm{~g}$. Attributable mortality from IA was $9.5 \%$. In the two patients who died, the daily dose of LAB was low at 1.5 and $3 \mathrm{mg}$. These are encouraging findings when compared with current literature figures of responses of $54 \%$ and attributable mortalities of at least 19\%. This does not prove that LAB resulted per se in a good outcome for the patients. It does suggest that the use of modestly high doses of LAB, given early as first-line treatment, in a setting of an experienced dedicated unit using optimal support measures, are strongly associated with a favourable outcome from IA.

We have to refine our diagnostics and explore ways of maximising options for treatment of IA. Meanwhile, it is hard to disprove the heightened efficacy of the lipid amphotericins, in particular LAB. Cost is a factor, but there is much to support the contention that, although drug acquisition costs are higher, overall final costs are lower and lives are saved. We should pursue what we believe to be the current best treatment option for our patients.

MICHAEL ELLIS Department of Medicine, Faculty of Medicine and Health Sciences, PO Box 17666, UAE University, Al Ain, Abu Dhabi, UAE

\section{References}

1. Denning DW, Marinus A, Cohen $\mathrm{J}$ et al. An EORTC multicentre prospective study of invasive aspergillosis in haematological patients: diagnosis and therapeutic outcome. $J$ Infect 1998; 37: 173-180.

2. Lin S, Schranz J, Teutsch SM. Aspergillosis case-fatality rate: systematic review of the literature. Clin Infect Dis 2001; 32: 358-366.

3. Bates DW, Su L, Yu DT et al. Mortality and costs of acute renal failure associated with amphotericin B therapy. Clin Inter Dis 2001; 32: 686-693.

4. Johansen HK, Gotzsche PC. Amphotericin B lipid soluble formulations vs amphotericin B in cancer patients with neutropenia. The Cochrane database of systematic reviews 2001; [3]: CD 000969.

5. Ellis M. Amphotericin B preparations: a maximum tolerated dose in severe invasive fungal infections? Transplant Infect Dis 2000; 2: 51-61.

6. Allende MC, Lee JW, Francis $\mathrm{P}$ et al. Dose-dependent antifungal activity and nephrotoxicity of amphotericin B colloidal dispersion in experimental pulmonary aspergillosis. Antimicrob Agents Chemother 1994; 38: 518-522. 
7. Leenders ACAP, de Marie S, Ten Kate MT, Bakker-Wondenberg IAJM, Verbrugh HA. Liposomal amphotericin B [AmBisome] reduces dissemination of infection as compared with amphotericin B deoxycholate [Fungizone] in a rat model of pulmonary aspergillosis. J Antimicrob Chemother 1996; 38: 215-225.

8. Prentice HG, Hann IM, Herbrecht $\mathrm{R}$ et al. A randomized comparison of liposomal versus conventional amphotericin $\mathrm{B}$ for the treatment of pyrexia of unknown origin in neutropenic patients. Br J Haematol 1997; 98: 711-718.

9. Walsh TJ, Finberg RW, Arndnt $\mathrm{C}$ et al. Liposomal amphotericin B for empirical therapy in patients with persistent fever and neutropenia. N Engl J Med 1999; 340: 764-771.

10. Leenders ACAP, Daenen S, Jansen RLH et al. Liposomal amphotericin B compared with amphotericin B deoxycholate in the treatment of documented and suspected neutropeniaassociated invasive fungal infections. $\mathrm{Br} J$ Haematol 1998; 103: 205-212.

11. Wingard JR, White MH, Anaissie EJ et al. A randomized double blind safety study of AmBisome and Abelcet in febrile neutropenic patients. Focus on Fungal Infections, San Diego 1999. Abstract no 15.

12. Francis P, Lee JW, Hoffman A et al. Efficacy of unilamellar liposomal amphotericin B in treatment of pulmonary aspergillosis in persistently granulocytopenic rabbits: the potential role of bronchoalveolar D-mannitol and serum galactomannan as markers of infection. J Infect Dis 1994; 169: 356-368.

13. Ellis ME, Spence D, de Pauw B et al. An EORTC international multicenter trial [EORTC number 19923] comparing two dosages of liposomal amphotericin B for treatment of invasive aspergillosis. Clin Infect Dis 1998; 27: 1406-1412.

14. van Etten EWM, ten Kate MT, Stearne LET, BekkerWondenberg IAJM. Amphotericin B liposomes with prolonged circulation in blood: in vitro antifungal activity, toxicity, and efficacy in systematic candidiasis in leukopenic mice. Antimicrob Agents Chemother 1995; 39: 1954-1958.

15. Dodds ES, Drew RH, Perfect JR. Antifungal pharmacokinetics: review of the literature and clinical applications. Pharmacotherapy 2000; 20: 1335-1355.

16. Anaissie E, Paetznick, V, Proffitt R, Adler-Moore J, Bodey GP. Comparison of the in vitro antifungal activity of free and liposome-encapsulated amphotericin B. Eur J clin Microbiol Infect Dis 1991; 10: 665-668.

17. Adler-Moore JP, Proffitt RT. Development, characterization, efficacy and mode of action of AmBisome, a unilamellar liposomal formulation of amphotericin B. $J$ Liposome Res 1993; 3: 429-450.

18. NeXstar Pharmaceuticals Inc. AmBisome ${ }^{\mathbb{R}}$ [amphotericin B] liposome for injection: prescribing information. Deerfield, IL, August 1997.

19. Paterson PJ, Johnson EM, Ainscough S et al. Treatment failure in invasive aspergillosis due to poor tissue penetration by antifungal drugs. 40th International Conference on Antimicrobial Agents and Chemotherapy, Toronto, Canada, 2000: Abstract no. 1326.

20. Caillot D, Couaillier J-F, Bernard A et al. Increasing volume and changing characteristics of invasive pulmonary aspergillosis on sequential thoracic computed tomography scans in patients with neutropenia. J Clin Oncol 2001; 19: 253-259.

21. Caillott D, Casasnovas O, Bernard A et al. Improved management of invasive pulmonary aspergillosis in neutropenic patients using early thoracic computed tomographic scan and surgery. J Clin Oncol 1997; 15: 139-147.

22. Hughes WT, Armstrong D, Bodey GP et al. 1997 guidelines for the use of antimicrobial agents in neutropenic patients with unexplained fever. Clin Infect Dis 1997; 25: 551-573.

23. Adler-Moore J. AmBisome targeting to fungal infections. Bone Marrow Transplant 1994; 14 Suppl 5: S3-S7. 